\title{
Lung Inflammatory Myofibroblastic Tumor Carrying TGF -ROS1 Fusion: About a Case
}

\author{
Erraichi $\mathrm{H}^{1 *}$, Berrad $\mathrm{S}^{1}$, Nouiakh L ${ }^{1}$, Ammadour $\mathrm{L}^{1}$, Oualla $\mathrm{K}^{1}$, Benbrahim $\mathrm{Z}^{1}$, Arifi $\mathrm{S}^{1}$, Asmae \\ Mazti $^{2}$, Nawal Hammas ${ }^{2,3}$, Hinde El Fatemi ${ }^{2,3}$ and Mellas $\mathbf{N}^{1}$ \\ ${ }^{1}$ Medical oncology department, CHU Hassan II fes, Morocco. \\ ${ }^{2}$ Department of Anatomopathology, Hassan II University Hospital, Morocco \\ ${ }^{3}$ Biomedical and Translational Research Laboratory, Morocco
}

*Corresponding author: Hayat Erraichi, Medical oncology department, CHU Hassan II fes, Morocco

To Cite This Article: Erraichi H, Berrad S, Nouiakh L, Ammadour L, Oualla K, et al., Lung Inflammatory Myofibroblastic Tumor Carrying TGF -ROS1 Fusion: About a Case. Am J Biomed Sci \& Res. 2021 - 11(4). AJBSR.MS.ID.001649. DOI: 10.34297/AJBSR.2021.11.001649.

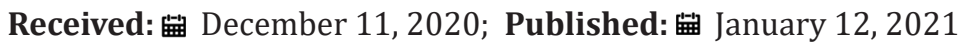

\begin{abstract}
Background: Inflammatory lung pseudotumors are extremely rare. Their pathogenesis is controversial, their diagnosis is often difficult, and their clinical behavior can be unpredictable.

Case présentation: This is a 48-year-old patient hospitalized for management of an intracranial hypertension syndrome whose radiological assessment was in favor of metastatic brain lesion with primary pulmonary origin, the biopsy with anatomopathological study posed the diagnosis of " an inflammatory myelofibroblastic tumor associated with a TGF-ROS 1 fusion ALK-negative, the patient benefited from treatment with a targeted therapy based on crizotinib with good clinical response, in particular the regression of symptoms and radiological response, patient currently still under treatment.
\end{abstract}

Conclusion: Immunohistochemistry for ROS1 helped to support the diagnosis of a subset of ALK negative inflammatory myofibroblastic tumors.

\section{Introduction}

Inflammatory myofibroblastic tumors (IMT) are rare mesenchymal neoplasms of borderline malignancy typically affecting children and young adults. We present an adult case of IMT of pulmonary localization with a ROS1 -TGF fusion treated with crizotinib

\section{Case Présentation}

48-year-old patient with a history of HP-induced gastritis, mitral valve replacement under anticoagulant, mitral ring in 1994, Mitral plasty by left lateral thoracotomy in 1982, former chronic tobacco user weaned.

Presented in the emergency room with a HTIC syndrome and partial convulsions of the left upper limb evolving in a context of weight loss estimated at $8 \mathrm{~kg}$ in 3 months $\mathrm{n}$ a brain scan was performed showed multiple brain injury processes, probably metastatic, the thoraco-abdomino-pelvic scanner revealed a lung parenchymal tumor process locally advanced 83mm LIG,
Osteocondansant lesions of the axial and peripheral spine, Scattered calcified pulmonary parenchymal micronodular lesions.

We completed with an MRI which demonstrated the presence of multiple tissue lesions, the largest of which was at the right frontal level measured at $22 \mathrm{~mm}$ with peri-lesional edema with diffuse cerebral edema, Similar right cerebellar lesion measured at $45 \mathrm{~mm}$ with peri-lesional edema and appearance. laminated V4. patient put under treatment with depakine 500mg $2 * /$ day, corticosteroid therapy and subsequently a surgical biopsy of the pulmonary lesion was performed with pathological results in favor of a chronic inflammatory fibrosis change without specificity hence the realization of a stereotaxic biopsy of the cerebral metastasis, the histological appearance of which was compatible with an inflammatory cerebral pseudo tumor and the immunohistochemistry confirms the diagnosis by the positivity of CD138 + and the negativity of Anti GFAP, an expert opinion was also requested from BERGONIE in France which confirms the 
hypothesis of a myelofibroblastic tumor inf lammatory combined with TGF-ROS1 fusion treatment with crizotinib $200 \mathrm{mg}$ twice daily.

The control scanner after 3 months showed a partial radiological response according to the criteria for the evaluation of the response in Solid tumors (RECIST), so the patient also reported clinical improvement

\section{Discussion}

Inflammatory myofibroblastic tumor (IMT) is a rare mesenchymal tumor that belongs to a subtype of soft tissue sarcoma, with an overall prevalence of approximately $0.04 \%$ to $0.7 \%$ [1]. They can occur at any age; however, it is more common in children and adolescents. The lungs are the most common site of onset of IMT, although it can also occur at several sites such as the retro peritoneum, abdomen, and pelvic cavity [1]. Common symptoms include cough, pain and fever, although up to $70 \%$ of patients are asymptomatic $[2,3]$.

Imaging is nonspecific and often shows a solitary and wellcircumscribed peripheral mass. Pathologically, these tumors are composed of a heterogeneous population of inflammatory and mesenchymal cells [3,4]. immunohistochemically, TMI expresses vimentin (95 to 100\%) and desmin (5 to 80\%); these two markers are expressed in our patient. It also expresses AML (48 to 100\%) and keratin (10 to 89\%). It is negative for skeletal muscle markers, namely myoglobin, myogenin and MyoD1 $[5,6]$.

Definitive diagnosis of IPT can therefore be difficult with needle biopsies, even with multiple attempts [3,4]. Important differential diagnosis considerations include sarcoma, malignant fibrous histiocytoma, lymphoma, and malignant plasmacytoma [7]. Initially Surgery was the most important therapeutic approach for IMT because the tumor is unresponsive to radiotherapy and chemotherapy; thus, the treatment options for patients with unresectable IMT are very limited [1].

However, IMT has regained attention in recent years due to its molecular characteristics and actionable goals. Here, we reported a 42-year-old patient who was diagnosed with ALK-negative metastatic lung IMT based on IHC and FISH tests. NGS-based broad genomic profiling showed the patient to have a TFG-ROS1 rearrangement.

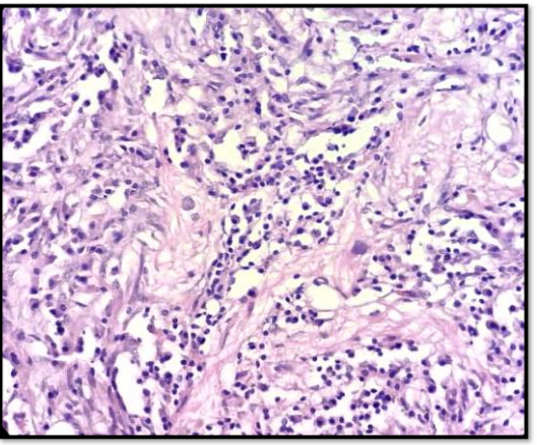

Figure 1: Spindled myofibroblasts and fibroblasts without atypia, surrounded by the inflammatory cells (HESx40)

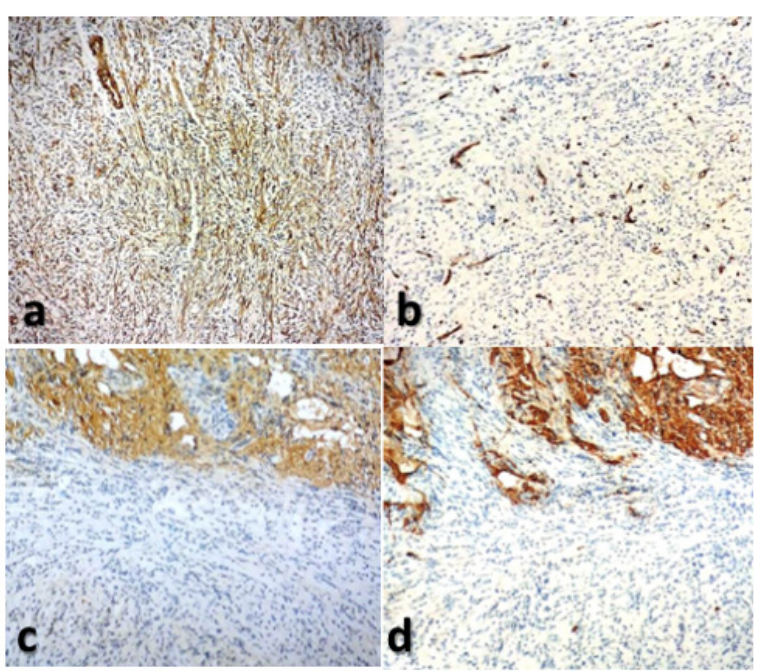

Figure 2: Spindle cells with diffuse cytoplasmic staining for SMA (a). Negative staining for CD34 (b), PS100 (c) and GFAP (d). 
About $50 \%$ of inflammatory myofibroblastic tumors harbor rearrangements of the ALK gene and are positive for ALK by immunohistochemistry $[8,9]$. ALK is therefore a useful marker to confirm the diagnosis of this type of tumor. However, the diagnosis of ALK negative inflammatory myofibroblastic tumors remains a significant challenge [8]. When tumors appear in pediatric and adolescent patients in the lung or abdomen and exhibit cytoarchitectural features Typical (i.e., uniform spindle cell bundles with vesicular nuclei, mixed with plasma cells and lymphocytes), the diagnosis of inflammatory myofibroblastic tumor is relatively straightforward even in the absence of ALK expression. In contrast, when such tumors occur in older patients or in unusual anatomical sites (eg, somatic soft tissue), or exhibit notable nuclear atypia, more aggressive spindle cell sarcomas may be serious diagnostic considerations. In recent years, a considerable proportion of ALKnegative IMT patients have been shown to harbor rearrangements in various genes, including ROS1, NTRK, PDGFR $\beta$ and RET. Effective targeted therapies are available for patients with these rearrangements [10].

This report expands the clinicopathologic spectrum of ROS1 rearranged IMT by documenting an adult example, which occurred in the lung. Only 3 cases of IMT with TFG - ROS1 have ever been reported in the literature, and these tumors appeared in the lung, mesentery and stomach of a 6year old child, an 8year old boy and a 30year old adult, respectively. Therefore, identification of the TFG ROS1 fusion in this case not only contributed to precise molecular subtyping, but also indicated treatment with targeted molecular therapy with crizotinib.

\section{Conclusion}

The report of this myelofibroblastic tumor case showed that immunohistochemistry for ROS1 may be useful in supporting the diagnosis of a subset of ALK negative inflammatory myofibroblastic tumors and may select some clinically aggressive and metastatic cases for targeted therapy directed against ROS1.

\section{Ethics Approval and Consent to Participate}

Not applicable

\section{Consent for Publication}

Consent from patient was obtained before publication of this case report.

\section{Availability of Data and Materials}

The data are available from the corresponding author on reasonable request.

\section{Competing Interests}

The authors declare that they have no competing interests.

\section{Funding}

None.

\section{References}

1. Panagiotopoulos N, Patrini N, Gvinianidze L, Woo WL, Borg E, et al. (2015) Inflammatory myofibroblastic tumour of the lung: a reactive lesion or a true neo- plasm? J Thorac Dis 7(5): 908-911.

2. Melloni G, Carretta A, Ciriaco P, Arrigoni G, Fieschi S, et al. (2005) Inflammatory pseudotumor of the lung in adults. Ann Thorac Surg 79: 426-432.

3. Kotoulas C, Konstantinou M, Fotinou M, Triggidou R, Papamichalis G, et al. (2006) Inflammatory pseudotumor of the lung: Our experience. Pneumon 19(1): 54-58.

4. Ishida T, Oka T, Nishino T, Tateishi M, Mitsudomi T, et al. (1989) Pseudotumeur inflammatoire du poumon chez l'adulte: analyse radiographique et clinicopathologique. Ann Thorac Surg 48: 90-95.

5. Cheng L, Foster SR, MacLennan GT, Lopez-Beltran A, Zhang S, et al. (2008) Inflammatory myofibroblastic tumors of the genitourinary tractsingle entity or continuum? J Urol 180(4): 1235-1240.

6. Lott S, Lopez-Beltran A, Montironi R, Montironi R, Chenget L, et al. (2007) Soft tissue tumors of the urinary bladder, part I: myofi- broblastic proliferations, benign neoplasms, and tumors of uncertain malignant potential. Hum Pathol 38(6): 807-823.

7. Gleason BC, Hornick JL (2008) Inflammatory myofibroblastic tumours: where are we now? J Clin Pathol 61(4): 428-437.

8. Coffin CM, Patel A, Perkins S, Elenitoba-Johnson KS, Perlman E, et al. (2001) ALK1 and p80 expression and chromosomal rearrangements invol- ving 2 p23 in inflammatory myofibroblastic tumor. Mod Pathol 14(6): 569-576.

9. Lovely CM, Gupta A, Lipson D, Otto G, Brennan T, et al. (2014) Inflammatory myofibroblastic tumors harbor multiple potentially actionable kinase fusion. Cancer Discov 4(8): 889-895.

10. Antonescu CR, Suurmeijer AJ, Zhang L, Sung YS, Jungbluth AA, et al. (2015) Molecular characterization of in- flammatory myofibroblastic tumors with frequent ALK and ROS1 gene fusions and rare novel RET rearrangement. Am J Surg Pathol 39(7): 957-967. 